\title{
Age-related responses of right ventricle in swim-trained rats: changes in lactate and pyruvate contents and lactate dehydrogenase activity.
}

\author{
V. Anitha, S. Asha Devi* \\ Department of Zoology, Bangalore University, Bangalore-560 056, India \\ Received 15 November 1995; revised 3 May 1996; accepted 8 May 1996
}

\begin{abstract}
Age related changes in carbohydrate substrates such as, glucose, glycogen, pyruvic acid and lactic acid and the activity of lactate dehydrogenase (LDH) and LDH isoenzyme profile were evaluated in the right ventricle (RV) of swim-trained rats of 6- (adult), 12- (middleaged) and 18- (old) months-of-age. Moderate hypertrophy was seen in the heart and RV in response to training in all age groups with the 12 months exhibiting a significant increase. While resting levels of pyruvate and glucose in the RV showed small elevations in adult and middle-aged rats, lactic acid showed reductions in all ages. Glycogen supercompensation was seen in the RV of trained animals. These age-related alterations in RV were associated with decreases in blood lactic acid and glucose in the trained rats belonging to all ages. Total protein of the RV decreased with age and exercise increased the content. Total LDH and $\mathrm{M}_{4}-\mathrm{LDH}$ activities decreased with age. However, training increased their activities in all ages. These changes in the RV suggests that swimming activity produces adaptations (e.g. increased $\mathrm{LDH}$ and $\mathrm{M}_{4}$ ) in all age groups. Considering the degree of adaptations, it can be suggested that adult and middle-aged are suitable for initiating swim-training programs, but not in old age.
\end{abstract}

* Corresponding author. Tel.: + $9180355036 / 214$.

0047-6374/96/\$15.00 (C) 1996 Elsevier Science Ireland Ltd. All rights reserved PII S0047-6374(96)01749-6 
Keywords: Lactate: Lactate dehydrogenase; Isoenzyme; Ageing; Exercise: Right Ventricle; Rat

\section{Introduction}

It is well known that exercise reflects physical activity and demands a greater supply of energy as compared to sedentary animals. Different types of exercises, swimming and running elicit varied responses from various substrates, such as glucose, lactate and pyruvate, in the blood and this may be due to changes in the effect of stress imposed on the individual organs $[1,2]$. The cardiac muscle has been a tissue of immense interest due to the fact that it needs an uninterrupted and unfailing energy supply and has a predominant aerobic metabolism, the metabolic fuels being fatty acids, glucose, lactate, pyruvate and ketone bodies. Several conflicting reports have stemmed from the resting levels of blood glucose being decreased [3] or unchanged [4]. Lactic dehydrogenase (LDH), a key glycolytic enzyme is reported to increase in the heart during exercise training in rats [5] and is related to cardiac hypertrophy $[5,6]$.

The above mentioned studies provide no information on the substrates, $\mathrm{LDH}$, and LDH isoenzymes of the right ventricle (RV) under conditions of physical training in rats. The RV has a significantly greater volume fraction of myofibrils with a lesser number of coronary capillaries/myofibre when compared to the left ventricle (LV) [7]. Studies on age-related responses of the RV to physical training may prove extremely informative due to differences in the two regions of the ventricle. The young and old ventricular muscle may combat the anaerobicity induced by exercise training with less or comparable vigour.

This paper is concerned with the glucose, lactate, glycogen and pyruvate content in the hypertrophied RV along with LDH and its isoenzymes. These substrates which are definitive indicators of anaerobic glycolytic metabolism are investigated in the aging ventricle. Attempts are made to determine a suitable age at which exercise can be initiated in order to derive the benefits of training.

\section{Materials and methods}

\subsection{Chemicals}

Sodium lactate, acrylamide, bis, ammonium per sulphate, NADH, NAD, nitroblue tetrazolium, phenazine methosulphate, sodium pyruvate, LDH isotrol, bromophenol blue, lithium lactate and bovine serum albumin were purchased from Sigma (St. Louis, MO). All other biochemicals were of reagent grade. 


\subsection{Animal care and training program}

Male pathogen-free albino rats of Wistar strain were purchased from the Central Animal Facility, Indian Institute of Science, Bangalore, India. These animals of 6-months (adults), 12-months (middle-aged) and 18-months (old) were housed three per cage in polypropylene cages $(30 \times 22 \times 14 \mathrm{~cm})$ in a room maintained at $27^{\circ} \mathrm{C} \pm 2{ }^{\circ} \mathrm{C}$ with a $12 \mathrm{~h}$ light $/ 12 \mathrm{~h}$ dark cycle. They had free access to tap water and a standard commercial diet (Lipton India, Calcutta). In this investigation we allocated rats to three groups: (A) trained swimmers (SW-T); (B) untrained swimmers (SW-U); and (C) sedentary controls (SE-C). SW-T were made to exercise initially for a 5 min bout twice a day and the duration was increased by 5 min daily until they were swimming for a $30 \mathrm{~min}$ bout twice a day without any exhaustion. Animals swam in a glass tank $(77 \times 39 \times 38 \mathrm{~cm})$ at $34^{\circ} \mathrm{C} \pm 1{ }^{\circ} \mathrm{C}$ at a depth of $22 \mathrm{~cm}$ for the 6-months and at $27 \mathrm{~cm}$ for the 12- and 18-months-old. SW-U were allowed to swim for a $30 \mathrm{~min}$ bout twice in $24 \mathrm{~h}$ before sacrifice, along with the SW-T and SE-C. During training, the rats were carefully observed for any symptoms of fatigue and any pathological conditions. Under such circumstances exercise was immediately terminated. All animals were sacrificed by etherization.

\subsection{Preparation of tissues}

For the biochemical estimations the abdomen and chest were opened by a midline incision and the blood was collected by cardiac puncture into $\mathrm{NaF}$ containing tubes. The heart was perfused with ice-cold buffered saline. The organ was excised, trimmed of blood vessels and connective tissue. RV, LV and atria (A) were separated and rinsed several times in ice-cold saline. RV was blotted free of any fluid using Whatman Filter paper No. 40, weighed and processed for the extraction and determination of biochemical parameters.

\subsection{Assays}

Glucose was measured using the method of Somogyi-Nelson [8]. The filtrates obtained from the deproteinized blood and tissue extract were heated with an alkaline copper reagent and then treated with a special arsenomolybdate colour reagent. Glucose concentrations were measured using glucose standards. Glycogen was extracted and quantitatively determined by the Anthrone method of Seifter et al. [9].

Pyruvic acid was assayed by the method of Friedman and Haugen [10]. Deproteinized samples were treated with 2,4-DNPH forming pyruvic hydrazone which reacts with a strong alkali to form a reddish compound which was measured with sodium pyruvate as standard.

Lactic acid was quantitated by the method of Barker and Summerson [11]. The glucose and other interfering material of the protein-free filtrates were removed and an aliquot of this was heated with concentrated sulphuric acid to convert lactic acid to acetaldehyde which was then determined by reaction with $p$-hydroxyphenyl in the presence of copper ions and using lithium lactate as standard. 
Total protein of the right ventricle was determined by the method of Lowry et al. [12] using bovine serum albumin as the standard.

LDH (E.C.1.1.1.27) was measured by the method of Cabaud, Wroblewski and Ruggieri [13]. Pyruvic acid was used, in the presence of $\mathrm{NADH}_{2}$ as the substrate. 2,4-DNPH was added and the hydrazone formed was determined using a standard prepared from pyruvate buffer.

Isoenzymes of $\mathrm{LDH}$ were separated by disc gel electrophoresis [14] on polyacrylamide gels. Gels were scanned in a Chromoscan (Joyce and Loebl, Gateshead). The area under each peak was measured for the activity of each isoenzyme and was represented as percent total area.

\subsection{Statistical methods}

All data are presented as means \pm standard deviation. The Student's $t$-test was applied to test the significance of variables. A value $P<0.001$ was considered highly significant. Changes in heart weight in the sedentary controls and swimtrained rats were analyzed by Karl Pearson correlation coefficient $(r)$ [15].

\section{Results}

\subsection{Heart weight}

Table 1 shows the pattern of weight changes in the different regions of the heart with aging and in response to swim-training. Total heart weight increases with age $(r=0.992)$. Training induces an increase in weight in all the ages $(r=0.785)$. Although the total heart weight is consistently higher in all the trained animals, this difference reaches a peak of $34 \%$ by 12 -months-of-age and the extent was similar in the right and left ventricles. However the extent of increase in 18-months-old is significantly less.

\subsection{Blood}

Table 2 shows the pyruvate content as a function of age and swim-training. There is no change in the pyruvate concentrations with age and training in SW-T group of 6- and 18-months-old, except for the SW-U group of 6-months-old animals showing a significant incrcasc. However, the 12 -months-old exhibited an insignificant increase in the SW-U and SW-T groups.

Lactic acid decreases progressively with age (Table 2). Physical conditioning reduces the acid content significantly in the 6-months-old and to a lesser extent in 12-months-old rats. However 18-months-old show an increase on training. SW-U of all ages show decreases in lactic acid to a lesser extent than SW-T animals. Insignificant changes in blood glucose are seen with age (Table 2). Six-months-old SW-T show decreases in glucose while 12-months and 18-months-old show no effect of training. Untrained swimmers fail to show any response. 


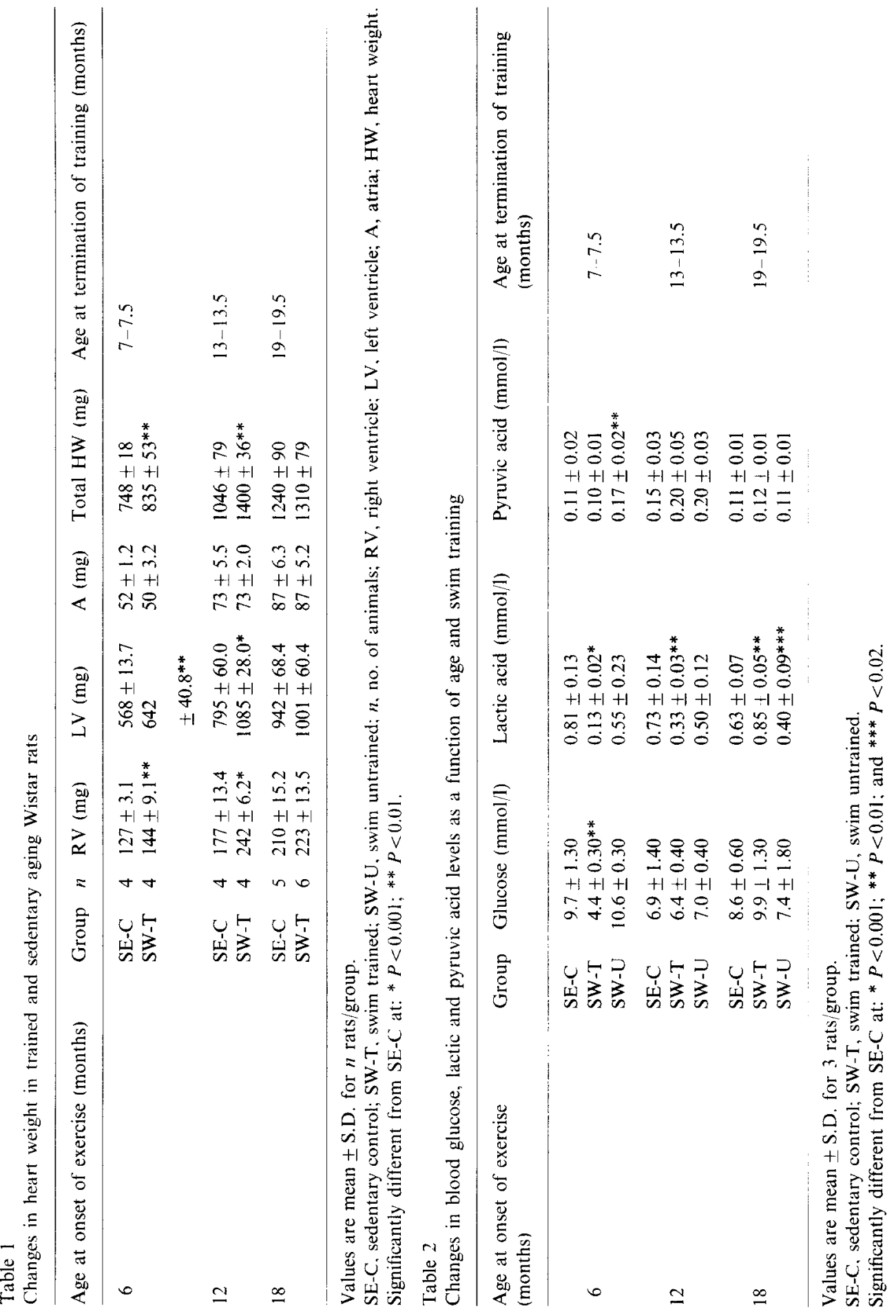




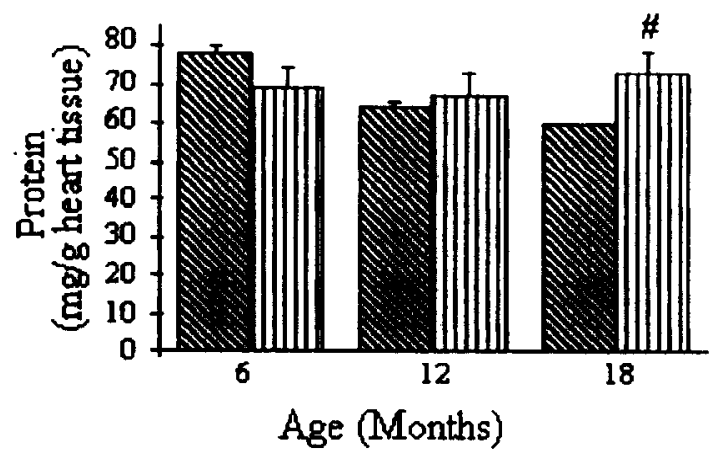

Fig. 1. Total protein content of the RV as a function of age and physical training. Values are mean \pm S.D. for three observations. \#, $P<0.01$ as compared to SE-C.

\subsection{Myocardium}

Fig. 1 shows the total protein content in the right ventricle with respect to age and exercise training. Protein decreases with the progression of age. Only 18months-old SW-T show significant elevation in protein content.

The pattern of variations in $\mathrm{RV}$ glucose and glycogen in response to training are represented in Table 3. Glycogen content varies little with age, the oldest group showing a decline by $34 \%$. SW-T show elevations of 44,50 and $56 \%$ in $6-, 12-$ and 18-months-old respectively. 12-months-old show significant elevation in glucose content in response to training.

Table 4 depicts the changes in lactic and pyruvic acid in the RV. Elevations in pyruvic acid is seen in $6-(23 \%)$ and $12-(31 \%)$ months-old in response to training.

Table 3

Age-related changes in cardiac glucose and glycogen content in response to swim training

\begin{tabular}{lllll}
\hline $\begin{array}{l}\text { Age at onset of exercise } \\
\text { (months) }\end{array}$ & Group & Glucose $(\mathrm{mg} / \mathrm{g})$ & Glycogen $(\mathrm{mg} / \mathrm{g})$ & $\begin{array}{l}\text { Age at termination of } \\
\text { training (months) }\end{array}$ \\
\hline \multirow{4}{*}{6} & SE-C & $1.0 \pm 0.20$ & $1.04 \pm 0.52$ & \\
& SW-T & $1.04 \pm 0.40$ & $1.44 \pm 0.14$ & $7-7.5$ \\
& SW-U & $1.0 \pm 0.25$ & $1.20 \pm 0.16$ & \\
& SE-C & $0.62 \pm 0.25$ & $1.0 \pm 0.05$ & \\
12 & SW-T & $1.35 \pm 0.15^{* * *}$ & $1.5 \pm 0.10^{*}$ & $13-13.5$ \\
& SW-U & $0.42 \pm 0.15$ & $0.8 \pm 0.10^{* * * *}$ & \\
& SE-C & $0.73 \pm 0.14$ & $0.66 \pm 0.20$ & \\
18 & SW-T & $0.62 \pm 0.00$ & $1.52 \pm 0.30^{* * *}$ & $19-19.5$ \\
& SW-U & $1.25 \pm 0.00^{* *}$ & $0.76 \pm 0.00$ & \\
\hline
\end{tabular}

Values are mean \pm S.D. for 3 rats/group.

SE-C, sedentary control; SW-T, swim trained; SW-U, swim untrained.

Significantly different from SE-C at: ${ }^{*} P<0.002 ;^{* *} P<0.01{ }^{* * *} P<0.02$; and ${ }^{* * * *} P<0.05$. 
Table 4

Age-related changes in cardiac lactic and pyruvic acid content in response to swim training

\begin{tabular}{lllll}
\hline $\begin{array}{l}\text { Age at onset of exer- } \\
\text { cise (months) }\end{array}$ & Group & Lactic acid $(\mathrm{mg} / \mathrm{g})$ & $\begin{array}{l}\text { Pyruvic Acid } \\
(\mathrm{mg} / \mathrm{g})\end{array}$ & $\begin{array}{l}\text { Age at termination } \\
\text { of training (months) }\end{array}$ \\
\cline { 1 - 2 } 6 & SE-C & $1.02 \pm 0.23$ & $0.13 \pm 0.01$ & \\
& SW-T & $0.026 \pm 0.00^{* *}$ & $0.16 \pm 0.01^{* * * *}$ & $7-7.5$ \\
& SW-U & $0.82 \pm 0.54$ & $0.13 \pm 0.00$ & \\
12 & SE-C & $0.54 \pm 0.09$ & $0.16 \pm 0.01$ & \\
& SW-T & $0.052 \pm 0.00^{*}$ & $0.21 \pm 0.08$ & $13-13.5$ \\
& SW-U & $0.26 \pm 0.18$ & $0.12 \pm 0.00^{* * *}$ & \\
18 & SE-C & $0.52 \pm 0.00$ & $0.10 \pm 0.01$ & \\
& SW-T & $0.26 \pm 0.07^{* * *}$ & $0.06 \pm 0.00^{* * *}$ & $19-19.5$ \\
& SW-U & $0.45 \pm 0.10$ & $0.10 \pm 0.02$ & \\
\hline
\end{tabular}

Values are mean \pm S.D. for 3 rats/group.

SE-C, sedentary control; SW-T, swim trained; SW-U, swim untrained.

Significantly different from SE-C at: ${ }^{*} P<0.001$ : $^{* *} P<0.002$; $* * * P<0.01$; and ${ }^{* * * *} P<0.05$.

However, the 18-months-old show a decrease $(40 \%)$ in pyruvic acid content. Lactic acid depletion is seen in trained 6- $(97 \%), 12-(84 \%)$ and $18-(50 \%)$ months-old. However, the extent of reduction is less amongst the untrained rats of all ages.

Fig. 2 illustrates the changes in LDH activity as a function of age and training. LDH decreases with age. Swim-training induces elevations in activity, the old exhibiting the least increase. Fig. 3 depicts the effectiveness of swim-training on the

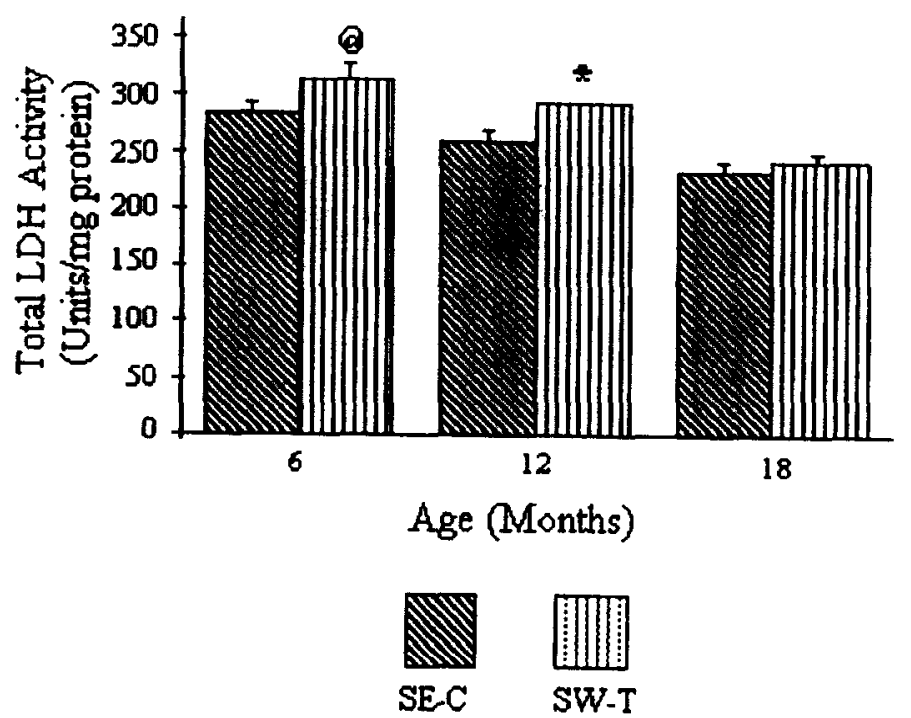

Fig. 2. Total $\mathrm{LDH}$ activity of the RV as a function of age and physical training. Values are mean \pm S.D. for three observations. $\star, P<0.001$; and $@, P<0.02$ as compared to SE-C. 

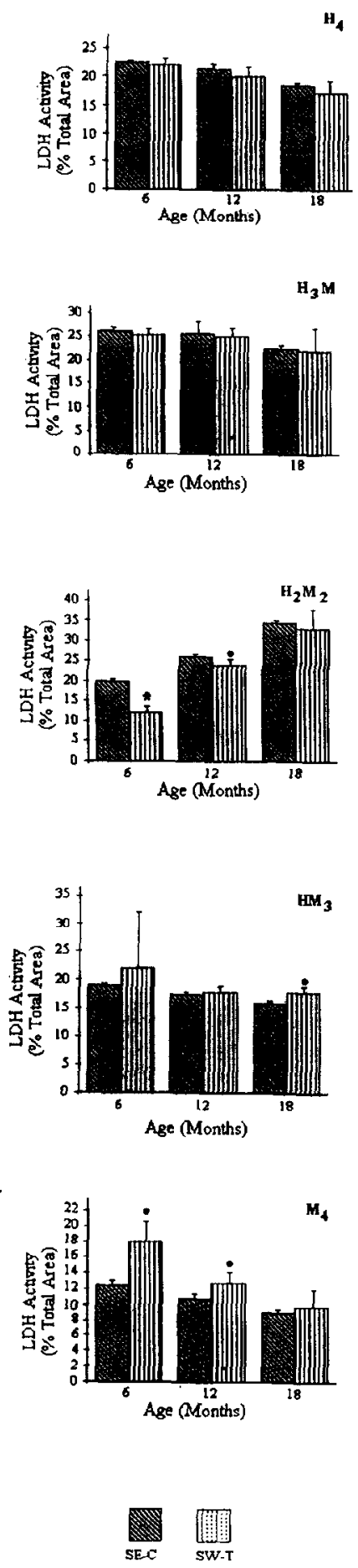

Fig. 3. LDH isoenzymes of the RV as a function of age and physical training. Activities are percent tot rrea \pm S.D. for three observations. $\star, P<0.001$; and $\bullet, P<0.05$ as compared to SE-C. 
$\mathrm{LDH}$ isoenzyme profile in aging rats. $\mathrm{M}_{4}-\mathrm{LDH}$ activity decreases with age along with $\mathrm{H}_{4}-$ and $\mathrm{H}_{3} \mathrm{M}-\mathrm{LDH}$. Training induces an increase in $\mathrm{M}_{4}$ activity in 6- and 12-months-old with the former exhibiting a greater elevation (44\%). 18-months-old fail to show any significant change in response to training.

\section{Discussion}

Physical training of rats by regular swimming is known to increase the heart weight if the exercise is sufficiently strenuous [16]. In the present study the mean total heart weight after four weeks of training was elevated in all three age groups, indicating that the training regimen was stressful. However, other factors, such as exercise type (swimming versus running) and the age of the animal are extremely important since the extent of adaptations of cardiac muscle are quite different in old animals $[2,6,17]$. The results of this study show that during swim-training resulting in cardiac hypertrophy, the LV and RV increase their weights equally. However, when exercise training was begun at 12-months-of-age there was a maximum hypertrophy of $34 \%$ in the ventricles. Our results of $6 \%$ elevation in LV, $\mathrm{RV}$ and total heart weight in 18-months-old swim-trained rats corroborate well with that reported by Rockstein et al. [16]. An equal extent of hypertrophy in LV and RV have been reported by York et al. [5] in their studies on 5-day-old swim-trained rats. In spite of morphometric differences in the two regions of the ventricles with $\mathrm{RV}$ having a smaller number of coronary capillaries/myofibre and reduced volume fraction of mitochondria than the LV [7], the extent of cardiac hypertrophy in terms of percent weight gain over respective controls were equal in the LV and RV. So, we went ahead with RV for all further studies.

The resting levels of blood lactate in the trained 6-months-old was reduced to a greater extent than in the 12-months-old. However, in the 18-months-old blood lactic acid increased in the trained state. A similar trend of depletion in 2- and 12-months-old have been reported earlier for blood lactic acid [2]. Tan et al. [1] have reported decreased elevation of resting levels of blood lactic acid in swimtrained rats.

Lactate is a major substrate of the heart and is also produced during glycolysis and under normal circumstances it is further degraded via the TCA cycle for the production of ATP by oxidative phosphorylation. In the present study, hypertrophied RV revealed highest depletion in lactate in the trained 6-months-of-age. Lower levels of lactate in the RV of trained animals can be interpreted based on the reports of Favier et al. [18], as decreased rate of lactate formation in the skeletal muscles, which in turn is due to the smaller differences in the arteriovenous lactate across the exercising muscles [19]. 18-months-of-age exhibited low lactate content in the trained state. The results of this study clearly show that a program of swim-training in the adult, middle-aged and old resulted in moderate RV hypertrophy associated with depletion in lactate content.

These changes were accompanied with elevated $\mathrm{LDH}$ and $\mathrm{M}_{4}-\mathrm{LDH}$ activities in all ages. Six-months-of-age showed a better response through greater elevation of 
$\mathrm{LDH}$ and $\mathrm{M}_{4}$ activities. A similar trend of increases in $\mathrm{LDH}$ and $\mathrm{M}_{4}$ has been reported in hypertrophied hearts under varied conditions of stress [5,20]. A program of treadmill running or swimming has been reported to increase the myocardial activity of some glycolytic enzymes, LDH and pyruvate kinase [20]. Ji et al. [21] have demonstrated decreased $K_{m}$ in myocardium for the conversion of lactic acid to pyruvic acid, without any changes in $\mathrm{K}_{\mathrm{m}}$ for the reverse reaction in endurance training. Furthermore, Keul [22] has shown that lactate accounts for a bigger proportion of the oxidative substrate and is preferentially utilized during exercise in the trained individuals. Hence the increased total LDH activity, as seen in the RV in the present study, may contribute to enhanced utilization of lactate as proposed by Sobel et al. [23] and York et al. [5]. Under such circumstances an increase in $\mathrm{M}_{4}-\mathrm{LDH}$ activity in trained $\mathrm{RV}$ would suggest an aerobic adaptation rather than an anaerobic one. In the sedentary rats decrease in $\mathrm{M}_{4}-\mathrm{LDH}$ activity in old age may decrease the capacity of the RV to withstand anaerobic conditions, which may result in lack of sufficient energy. This correlates with the higher frequency of heart failure and an inability to withstand longer periods of muscular exercise in old agc [24]. It is noteworthy that the present study has revealed a greater extent of elevation in pyruvate content in the RV of trained 6- and 12-months-old rats. These changes were in parallel with elevated levels of glucose in the RV. Kaniulainen et al. [25] have reported that swim-trained rats have an increased rate of myocardial glucose uptake at rest and during exercise. In the present study the resting levels of blood glucose was significantly lower in the 6-months-old while in the 12-monthsold, the depletion was less significant. Our results on depletion in glucose content of blood in response to training is in accordance with that reported by Stallknecht et al. [3] and Tan et al. [1]. It is well known that the sensitivity to the effect of insulin which is responsible for decreases in blood glucose is increased after training [26]. Furthermore, it is noteworthy that the resting levels of insulin in plasma is lower than in the untrained ones [4]. The lower level of blood glucose in the trained adult and middle-aged rats could be due to increased sensitivity to insulin and not, as proposed by Stallknecht et al. [3] that the activation of the sympathetic nervous system in response to hypoglycaemia is reduced by physical conditioning.

Ventricular hypertrophy was also accompanied by the increased levels of resting glycogen in all the trained age groups. The extent of elevation was not age specific. A similar trend has been reported by Scheuer and Stezoski [27] in cardiac glycogen of swim-trained rats at rest. Such glycogen supercompensation in rats has been reported by Poland et al. [28] after training and these changes occur independently of blood glucose levels.

Our study show that there is a moderate hypertrophy in the RV which shows adaptation towards aerobic metabolism in the adult and middle-aged rats as evidenced by the analysis of resting levels of carbohydrate substrates, LDH and its isoenzymes. There is a definite indication that training can be initiated with positive responses in the middle-aged animals. But the training protocol has not been shown to elicit adequate response in the old animals which have probably found the training regimen more strenuous. Our findings would be substantiated by further age related studies on the RV lactate uptake, its clearance and LDH kinetics in 
swim-trained rats. Further study of the effect of training on the LV and RV dimensions would provide a better relation with the aerobic adaptive changes in the two regions of the aging heart.

\section{Acknowledgements}

We thank R. Bhagavathi for her technical assistance and also Kumar and Srinivas for secretarial assistance.

\section{References}

[1] N. Tan, K. Morimoto, T. Sugiura, A. Morimoto and N. Murakami, The effects of running training on the blood glucose and lactate in rats during rest and swimming. Physiol. Behav., 51 (1992) $927-931$.

[2] R. Bhagavathi and S. Asha Devi, Interaction of exercise and age on substrates of carbohydrate metabolism. Indian J. Exp. Biol., 31 (1993) 72-75.

[3] B. Stallknecht, M. Kjaer, K.J. Mikines, et al., Diminished epinephrine response to hypoglycemia despite enlarged adrenal medulla in trained rats. Am. J. Physiol., 259 (1990) R998-R1003.

[4] K.J. Rodnick, C.E. Mondon, W.L. Haskell, S. Azhar and G.M. Reaven, Differences in insulin induced glucose uptake and enzyme activity in running rats. J. Appl. Physiol., 68 (1990) 513-519.

[5] J.W. York. D.G. Penney, T.A. Weeks and P.A. Stagno, Lactate dehydrogenase changes following several cardiac hypertrophic stresses. J. Appl. Physiol., 40 (1976) 923-926.

[6] J.A. Chesky, S. Lafollette, M. Travis and C. Fortado, Effect of physical training on myocardial enzyme activities in aging rats. J. Appl. Physiol., 55 (1983) 1349-1353.

[7] E.D. Canale, G.R. Campbell, J.J. Smolich and J.H. Campbell, Morphometry of Cardiac muscle. In A. Oksche and L. Vollrath (eds.), Cardiac Muscle, Springer-Verlag, New York, 1986, pp. 56-58.

[8] M. Somogyi, Notes on sugar determination. J. Biol. Chem., 195 (1952) 19-20. In B.L. Oser (ed.), Hawk s Physiological Chemistry, Tata McGraw-Hill, New Delhi, 1965, pp. 1054.

[9] S. Seifter, S. Dayton. B Novie and E. Muntwyler, The estimation of glycogen with the anthrone reagent. Arch. Biochem, 25 (1950) 191-194.

[10] Freidmann and Haugen, J. Biol. Chem., 147 (1943) 415. In B.L. Oser (ed.). Hank's Physiological Chemistry., Tata McGraw-Hill. New Dclhi, 1965, pp. 1108.

[11] Barker and Summerson, J. Biol. Chem., 138 (1941) 535. In B.L. Oser (ed.), Hawkis Physiological Chemistry.. Tata McGraw-Hill, New Delhi, 1965, pp. 1102.

[12] O.H. Lowry, N.J. Rosebrough, A.L. Farr and R.J. Randall, Protein measurements with the folin-phenol reagent. J. Biol. Chem, 193 (1951) 265-275.

[13] Cabaud, Wroblewski and Ruggiero, Am. J. Clin. Pathol., 30 (1958) 234. In B.L. Oser (ed).. Haw's Physiological Chemistry.. Tata McGraw Hill, New Delhi, 1965, pp. 1127.

[14] A.A. Dietz and T. Lubrano, Separation and quantitation of lactate dehydrogenase isoenzymes by disc electrophoresis. Anal. Biochem., 20 (1967) 246-257.

[15] R.R. Sokal and E.J. Rolf, The Priniciples and Practice of Statistics in Biological Research, In Sokal and Rolf (eds.), Biometry, W.H. Freemann, New York, 1981, pp. 21-36.

[16] M. Rockstein, J.A. Chesky and T. Lopez, Effects of exercise on the biochemical aging of mammalian myocardium. J. Gerontol., 36 (1981) 294-297.

[17] S. Asha and E. Radha, Activity of alanine aminotransferase in serum and ventricular tissue of rats after exercise and as a function of age. Curr. Sci., 50 (1982) 404-405.

[18] R.J. Favier, S.H. Constable, M. Chen and J.P. Holloszy, Endurance exercise training reduces lactate production. I. Appl. Physiol., 6I (1986) 885-889. 
[19] J. Henriksson, Training induced adaptations of skeletal muscle and metabolism during submaximal exercise. J. Physiol, 270 (1977) 661-675.

[20] P.D. Gollnick, P.J. Struck and T.P. Bogyo, Lactic dehydrogenase activities of rat heart and skeletal muscle after exercise training. J. Appl. Physiol., 22 (1976) 623-627.

[21] L.L. Ji, F.W. Stratman and H.A. Lardy, Chronic exercise training alters kinetic properties of rat skeletal muscle and myocardial lactate dehydrogenase. FEBS Lett., 203 (1986) 297-300

[22] J. Keul, Myocardial metabolism in athletes. In B. Pernow and B. Saltin (eds.), Muscle Metabolism During Exercise, Plenum. New York, 1971, pp, 447-455.

[23] B.E. Sobel, P.D. Henry, B.J. Ehrlich and C.M. Bloor, Altered myocardial lactic dehydrogenase isoenzymes in experimental cardiac hypertrophy. Lab. Invest., 22 (1970) 23-27.

[24] M.S. Kanungo. Phenotypic changes during aging. In M.S. Kanungo (ed.), Genes and Aging, Cambridge University Press, New York, 1994, pp. 21-36.

[25] H.P. Kaniulainen, P. Virtanen, H. Ruskoaho and T.E. Takala, Training increases cardiac glucose during rest and exercise in rats. Am. J. Physiol., 257 (1989) H839-H845.

[26] R.D. Fell and J.O. Holloszy, Exercise training and glucose uptake by skeletal muscle in rats. $J$. Appl. Physiol., 55 (1983) $1393-1396$.

[27] J. Scheuer and S.W. Stezoski, Protective role of increased myocardial glycogen stores in cardiac anoxia in the rat, Circ. Res., 27 (1970) 835-839.

[28] J.L. Poland, C. Trowbridge and J.W. Poland, Substrate repletion in rat myocardium, liver and skeletal muscles after exercise. Can. J. Physiol. Phamacol., 58 (1980) 1229. 